\title{
Multidisciplinary Management of a Rare Case of a Huge Low- Grade Chondrosarcoma of the Lumbar Spine: A Case Report
}

\author{
Samuel Jason Rolando Tua* ${ }^{1}$, Hendy Rachmat Primana Lubis ${ }^{1}$, Agus Hadian Rahim ${ }^{2}$, \\ Ahmad Ramdan ${ }^{2}$, Herry Herman ${ }^{3}$ \\ ${ }^{1}$ Department of Orthopaedic and Traumatology, Resident, Padjadjaran University, Bandung, West Java, Indonesia \\ ${ }^{2}$ Department of Orthopaedic and Traumatology, Spine Division, Padjadjaran University, Bandung, West Java, Indonesia \\ ${ }^{3}$ Department of Orthopaedic and Traumatology, Oncology Division, Padjadjaran University, Bandung, West Java, Indonesia
}

\section{ARTICLE INFO}

Received : 06 May 2020

Reviewed : 30 May 2020

Accepted : 14 August 2020

Keywords:

chondrosarcoma, oncology, spine,

resection, multidisciplinary

*Corresponding author:

Samuel Jason Rolando Tua

Departement Orthopaedic and

Traumatology, Padjadjaran University,

Bandung, West Java, Indonesia

samuel3101jason@yahoo.com

\section{ABSTRACT}

Introduction: Chondrosarcoma is the second most common primary bone sarcoma and arising in the spine. It is estimated to be $2 \%$ to $12 \%$ in incidence from various series. The use of neoadjuvant and adjuvant chemotherapy for chondrosarcomas is controversial. Low- and intermediate-grade chondrosarcomas respond poorly to chemotherapy. Although not much data on the efficacy of chemotherapy in the treatment of high-grade chondrosarcomas, it should be considered in any young patient with a high-grade tumor. Radiation is recommended when anything other than wide excision is performed for chondrosarcoma of any grade.

\begin{abstract}
Case Presentation: A 24-year old female presented to the clinic with a chief complaint of persistent low back pain and a lump in her back. Initially, the lump was the size of a tennis ball three years ago. At the time of the examination, the lump was at the size of a volleyball about $65 \times 63 \times 58$ centimeters. Systemic and general examinations were unremarkable. There were no comorbid conditions. From the results of the Clinicopathological Conference conducted by neurologists, pathologists, radiologists, orthopedic spine and oncology surgeons, it is advisable to conduct investigations such as x-rays, CT scans, MRI, biopsy, and surgery is recommended for evaluation of expansion of chondrosarcoma, evolving the spinal cord, and for resection of tumors.
\end{abstract}

Conclusion: Early diagnosis and complete resection of tumor and treatment of relevant symptoms represent a viable treatment for this rare disorder to achieve increased life expectancy, low recurrence of tumor, and improvement of quality of life.

\section{INTRODUCTION}

Chondrosarcoma is the second most common primary bone sarcoma. Subdivided into a variety of ways, histological grade, primary or secondary and/ or peripheral or central. The majority of the "conventional" chondrosarcomas are primary and occur between the ages of 30 and 50 years with the pelvis, femur, and shoulder girdle as the common sites of involvement. Histologically, chondrosarcomas are graded as I (low), II (intermediate), or III (high); according to the malignant appearance of the cells and the matrix produced, most are grade I or II. Radiologically, central chondrosarcoma presents as a well-defined lytic lesion with a narrow zone of transition and surrounding sclerosis with faint calcifications or with no sclerotic rim at all [1,2].

Chondrosarcomas arising in the spine is estimated to be $2 \%$ to $12 \%$ in incidence from various series [2], with the thoracic spine being the most frequent localization, followed by the cervical and lumbar region [3]. Unlike most other malignant spinal tumors, the lesions may arise in the vertebral body (5\%), the posterior elements $(40 \%)$, or both $(45 \%)$, since there are three growth centers in each vertebra from which the tumor may originate [4]. The most common presenting symptom in chondrosarcoma of the spine is pain; however, others may complain of a palpable mass or neurological deficits, which may manifest in half of the patients [3]. Chondrosarcoma is a mesenchymal tumor arising from cartilage cells. About $30 \%$ of skeletal system malignancies are chondrosarcomas [1], with less than $10 \%$ of predilection in the spine [2]. Chondrosarcoma is primarily common in patients aged 30 to 50 years. Men are affected more often than women [3]. Resistant to chemotherapy and radiotherapy [4], its most successful treatment is complete en bloc resection [5]. When en-bloc resection is not feasible, partial removal of the sarcoma followed by radiotherapy provides pain 
relief and improvement of neurological symptoms $[6,7]$. Low-grade (I) chondrosarcoma grows relatively slowly, and histological appearance is quite similar to the cells of that of normal cartilage with minimal aggressiveness, invasiveness, and metastatic potential. Grades II and III are increasingly faster-growing cancer cells with more anaplastic cellular characteristics and invasive to their surroundings. "Grade IV" is reserved for the most anaplastic, undifferentiated cartilage-derived tumor in some centers [8]

Low-grade chondrosarcomas or endochondrosarcomas may be treated by intralesional resection. Intermediateand high-grade chondrosarcomas, on the other hand, are treated by wide surgical resection. The use of neoadjuvant and adjuvant chemotherapy for chondrosarcomas is controversial. Low- and intermediategrade chondrosarcomas respond poorly to chemotherapy. Although not much data on the efficacy of chemotherapy in the treatment of high-grade chondrosarcomas, it should be considered in any young patient with a highgrade tumor. Radiation is recommended when anything other than wide excision is performed for chondrosarcoma of any grade.

\section{CASE PRESENTATION}

A 24-year old female presented to the clinic with a chief complaint of persistent low back pain and a lump in her back. Initially, the lump was the size of a tennis ball three years ago. At the time of the examination, the lump was at the size of a volleyball about $65 \times 63 \times 58$ centimeters (Figure 1). The back pain has prevented her from walking. Motor deficits were found during the examination; muscle strength of her left lower limb was decreased (4/5). Right lower limb strength was within normal limits (5/5). Bowel and bladder functions were normal. Systemic and general examinations were unremarkable. There were no comorbid conditions. From the results of the Clinicopathological Conference conducted by neurologists, pathologists, radiologists, orthopedic spine and oncology surgeons, it is advisable to conduct investigations such as $\mathrm{x}$-rays, computed tomography (CT) scans, magnetic resonance imaging (MRI), biopsy, and surgery is recommended for evaluation of expansion of chondrosarcoma, evolving the spinal cord, and for resection of tumors. The recommended examination is very important for the preparation of surgery to seeing the boundary of the tumor and its spread. The radiograph of the Lumbosacral spine shows shadows in the back and left side of the lumbar spine.

The CT scan of the abdomen shows a radiopaque inhomogeneous mass with calcification in the left flank region that appears to infiltrate subcutis, internal, and external oblique muscle, distract the pedicles, spinous, and transverse process of the 3rd, 4th, and 5th Lumbar vertebrae, and pushes the left kidney to a superoanterior location (Figure 2A).
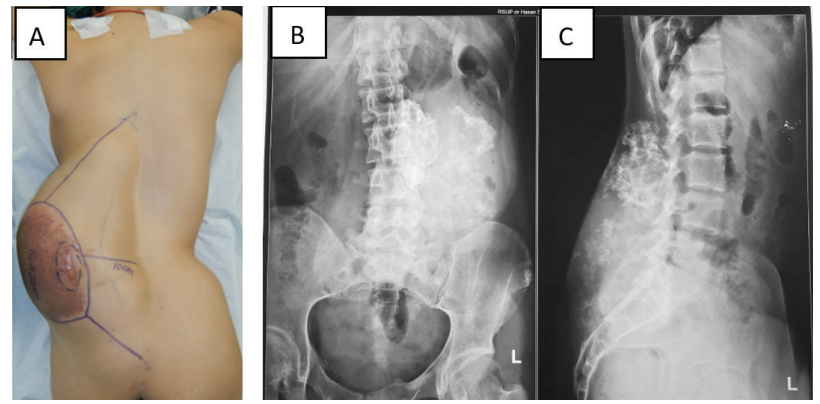

Figure 1. A. Clinical picture; Xray lumbosacral spine showing mass at Lumbar region (3rd to 5 th lumbar) from B. Anteroposterior view, and C. Lateral view.
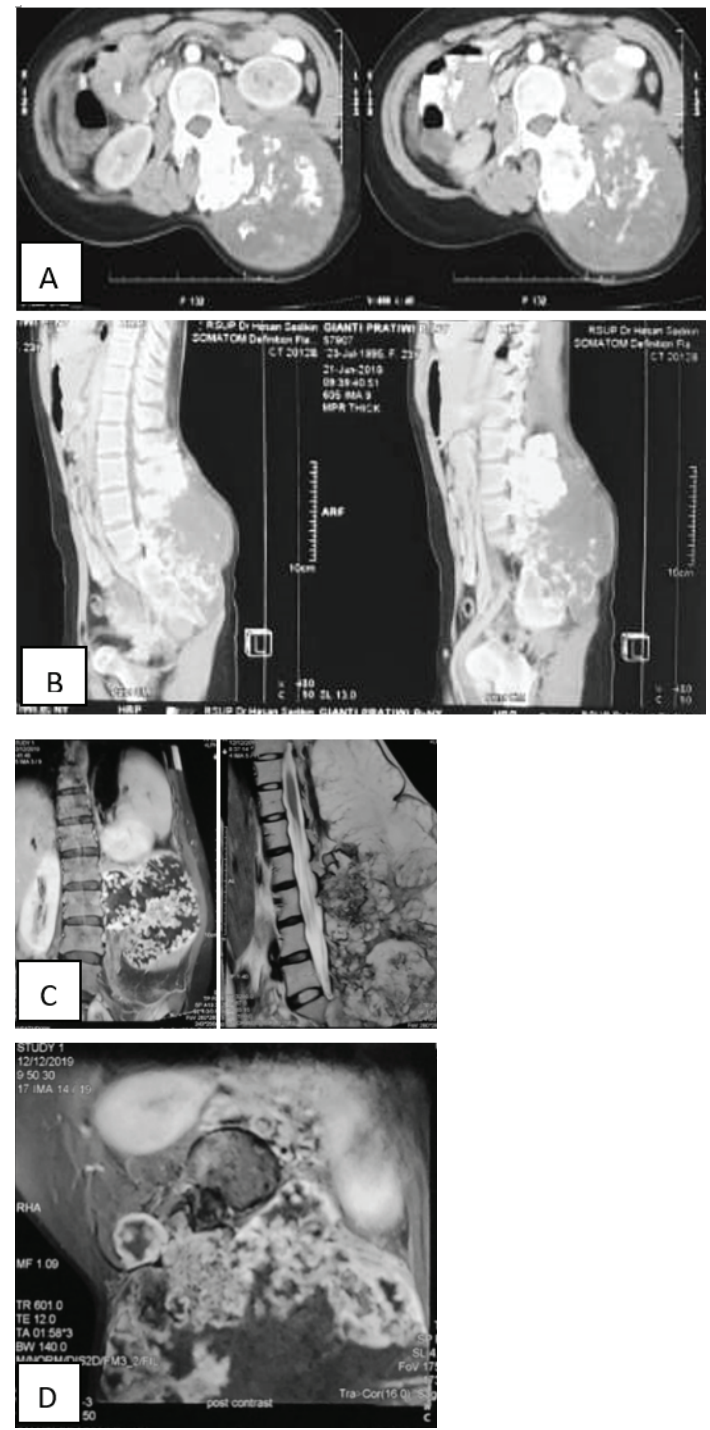

Figure 2. $C T$ Abdomen showing mass at $A$. 3rd Lumbar from axial view, B. 1st to 5th Lumbar from sagittal view. C. MRI Thoracolumbar shows mass at 3rd - 5th lumbar from Coronal view, and The mass apperars to spread to the spinous process in the 2 nd lumbar from sagittal view. D. Axial view of 3rd Lumbar shows mass from the spinous process to vertebra. 
Lumbosacral MRI examination shows a dense inhomogeneous mass with a possible chondroid matrix in the area behind the left posterior vertebrae extending from the thoracic to the lumbosacral region with infiltration of the subcutis, spinal muscles, iliocostal muscles, quadratus lumborum muscles, with the psoas muscle displaced anteriorly (Figure 2B). Biopsy of the tumor reveals a cartilaginous mass, with a round to oval, hyperplastic chondrocyte cells, mildly pleomorphic nucleus, and mitotic activity. A moderate amount of hypocellularity was found (Figure $\mathbf{3}$ ). The tumor produces a cartilaginous matrix; hence, a diagnosis of Low-grade chondrosarcoma is established.
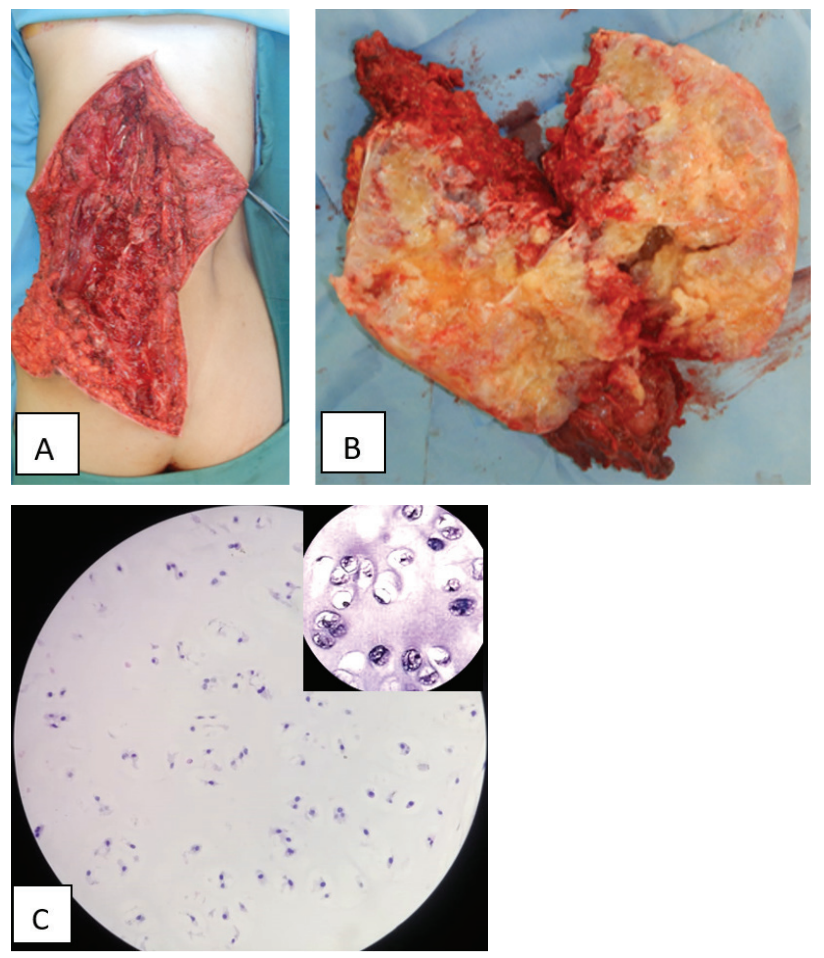

Figure 3. Intraoperative, A. After removing the mass from the lumbar vertebra, B. macroscopic view of the mass. C. Microscopic, Histopathological from biopsy and the result; a cartilaginous mass, with a round to oval, hyperplastic chondrocyte cells, mildly pleomorphic nucleus, and mitotic activity. A moderate amount of hypocellularity was found.

Surgical resection to completely remove the tumor was planned to prevent a recurrence. Chondrosarcoma is known to be highly resistant to chemotherapy and/ or radiotherapy. The spine was stabilized with a pedicle screw and rod as some of the posterior elements and ligaments were removed along with the tumors to maintain an oncology margin (Figure 4). The patient was stabilized for 3 days, during which the patient received blood transfusion and analgesics. Early mobilization was encouraged; the patient was discharged and followed up at the outpatient clinics for daily wound care and early weight-bearing and walking.
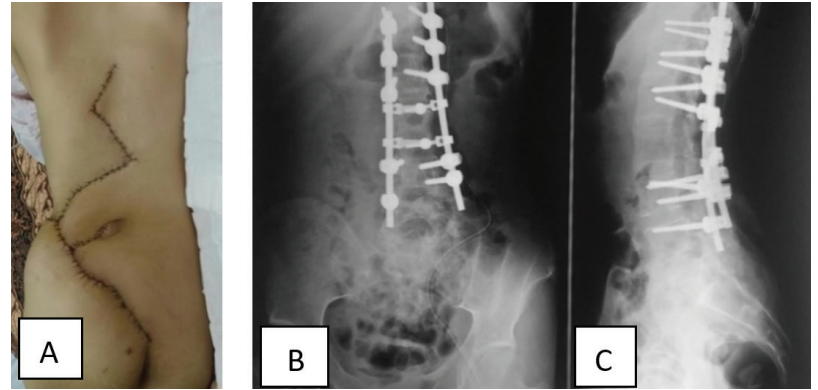

Figure 4. Postoperative: A. Clinical picture: surgical wound looks well maintained. Xray examination shows pedicle screw attached from thoracic to lumbar region from B. Anteroposterior view, C. lateral view.

The reevaluation of the removed tumor confirmed the diagnosis of well-differentiated low-grade chondrosarcoma. The surgery is performed using a posterior approach so that the oncology surgeon can evaluate the extent and expansion of the tumor to get a good resection margin in this area. Following a 13-month follow-up, the patient was able to bear weight and walk with $5 / 5$ muscle power on the right and left lower limbs. She underwent regular follow-up for early detection of recurrence with no visible involvement or extension for lamina or pedicle.

\section{DISCUSSION}

We report here a Chondrosarcoma involving a 24-yearold female. In terms of sexual preferences, chondrosarcoma is common for both sexes with male predominance [9]. Shives et al. [10] described a cohort of 20 patients from Mayo Clinic showing that men were most commonly affected. Chondrosarcoma is a malignant cartilage-forming bone neoplasm that accounts for $10.0 \%$ of all primary bone tumors among adults [11]. Typically, a low-grade neoplasm can arise from a pre-existing cartilage lesion such as an osteochondroma or enchondroma [12].

Chondrosarcoma may develop in any part of the body; however, the most common sites are the pelvis, rib cage, arms (upper arm or humerus), shoulder blades, and legs (proximal femur in the thigh and the tibia in the shin) [13]. Chondrosarcomas may be found in the spine or skull; however, this is extremely rare. Most chondrosarcomas of the spine occur in the thoracic spine, and patients typically present in their middle age with complaints of back pain and/or neurological symptoms [14].

Chondrosarcoma of the spine may present diagnostic difficulties. Differential diagnoses include chondroblastic osteosarcoma, chondromatosis, chordoma, ganglioneuroma, ganglioneuroblastoma, or schwannoma [11]. Plain X-ray, CT Abdomen, or MRI without adjuvant 
supporting examination may be insufficiently accurate to diagnose chondrosarcoma cases with the aforementioned possible differential diagnoses. Ideally, a resected tumor sample requires histopathological confirmation to determine the type and grading of the tumor [15].

In the case of a large, markedly heterogeneous, well capsulated, slightly lobulated left paraspinal lesion in the lower dorsal aspect of the thoracolumbar spine was detected with an additional extension of the mass to the right neural arc. The MRI findings in our case were similar to a case report by Strike et al. [9] Notable bone destruction was found with a concurrent calcified paraspinal mass.

Surgical resection is the recommended treatment for chondrosarcoma of the spine by a posterior approach to examine the margin of the tumor, and this procedure makes it easy to perform a posterior stabilization procedure; these tumors are notoriously resistant to chemotherapy or radiation therapy. In this case, by using the posterior approach, the oncology surgeon can examine the tumor at the position on the posterior side, especially the spinous process, lamina, spinous process, and paraspinal, so they can remove the tumor from the spine. The posterior approach involves excision of the tumor and the posterior element, which allows hemostasis of the epidural venous plexus, division of the posterior longitudinal ligament and annular fibrosis, evaluation during surgery, and makes it easy to carry out posterior stabilization so as not to injure the spinal nerve by a spine surgeon. The tumor was completely removed achieving a sufficient negative margin to avoid recurrence and, hopefully, provide disease-free survival.

Similar studies by Sundaresan et al. [2] and York et al. [13] found a significant increase in the disease-free interval after gross resection of the tumor versus subtotal excision. Local recurrence was noted in one of five patients who had a total resection with an average time to recurrence of 5.4 years, while nine of 13 patients with subtotal tumor excisions had disease recurrence at an average of 3.7 years [14]. The resection of huge chondrosarcoma requires the collaboration of oncologic orthopedic surgeons to achieve a reasonable negative margin. Additionally, the spine surgeon was tasked to manage spine instability caused by the wide resection of the tumor.

Following total resection of the tumor, the specimen was sent in multiple segments to two diagnostic facilities for histopathologic assessment, both in agreement for a diagnosis of a well-differentiated (low grade) chondrosarcoma. At the 13-month follow-up, the patient's conditions improved significantly, being able to stand and walk. Her muscle power of the lower limbs became $5 / 5$, and she could walk without difficulties. She is under regular follow-up for further evaluation.

\section{CONCLUSIONS}

Early diagnosis and complete resection of tumor and treatment of relevant symptoms represent a viable treatment for this rare disorder to achieve increased life expectancy, low recurrence of tumor, and improvement of quality of life.

\section{DECLARATIONS}

\section{Competing of Interest}

No potential conflict of interest relevant to this article was reported.

\section{Acknowledgment}

Not Applicable

\section{REFERENCES}

1. Gelderblom H, Hogendoorn PC, Dijkstra SD, et al. The clinical approach towards chondrosarcoma. Oncologist. 2008;13(3):320-9.

2. Sundaresan N, Rosen G, Boriani S. Primary malignant tumors of the spine. Orthop Clin North Am. 2009;40(1):21-36.

3. Tessitore E, Burkhardt K, Payer M. Primary clear-cell chondrosarcoma of the cervical spine. Case illustration. J Neurosurg Spine. 2006;4(5):424.

4. Knoeller SM, Uhl M, Gahr N, et al. Differential diagnosis of primary malignant bone tumors in the spine and sacrum. The radiological and clinical spectrum: minireview. Neoplasma. 2008;55(1):16-22.

5. Bergh P, Gunterberg B, Meis-Kindblom JM, Kindblom LG. Prognostic factors and outcome of pelvic, sacral, and spinal chondrosarcomas: a center-based study of 69 cases. Cancer. 2001;91(7):1201-12.

6. Boriani S, De lure F, Bandiera S, et al. Chondrosarcoma of the mobile spine: report on 22 cases. Spine. 2000;25(7):804-12.

7. Li $\mathrm{YH}$, Yao $\mathrm{XH}$. Primary intradural mesenchymal chondrosarcoma of the spine in a child. Pediatr Radiol. 2007;37(11):1155-8.

8. Prevedello DM-S, Cordeiro JG, Koerbel A, et al. Management of primary spinal chondrosarcoma: report of two cases causing cord compression. Arq Neuro-Psiquiatr. 2004;62:875-8.

9. Strike SA, McCarthy EF. Chondrosarcoma of the spine: a series of 16 cases and a review of the literature. lowa Orthop Journal. 2011;31:154-9.

10. Shives TC, McLeod RA, Unni KK, Schray MF. Chondrosarcoma of the spine. J Bone Joint Surg. 1989;71(8):1158-65. 
11. Lloret I, Server A, Bjerkehagen B. Primary spinal chondrosarcoma: radiologic findings with pathologic correlation. Acta Radiol. 2006;47(1):77-84.

12. Smith J, Ludwig RL, Marcove RC. Sacrococcygeal chordoma. A clinicoradiological study of 60 patients. Skeletal Radiol. 1987;16(1):37-44.

13. York JE, Berk RH, Fuller GN, et al. Chondrosarcoma of the spine: 1954 to 1997. Journal Neurosurg. 1999;90(1 Suppl):73-8.
14. Panelos J, Voulgaris S, Michos E, et al. Chondrosarcoma of the spine: A rare case with unusual presentation. Diagn Pathol. 2006;1(1):39.

15. Bishop MW, Somerville JM, Bahrami A, et al. Mesenchymal Chondrosarcoma in Children and Young Adults: A Single Institution Retrospective Review. Sarcoma. 2015;2015:608279. 